\title{
ASYMPTOTICS OF PROLATE SPHEROIDAL WAVE FUNCTIONS
}

\section{T. M. DUNSTER}

Abstract. Uniform asymptotic approximations are obtained for the prolate spheroidal wave functions, for both the angular functions $\operatorname{Ps}_{n}^{m}\left(x, \gamma^{2}\right)(-1<x<1)$ and radial functions $P s_{n}^{m}\left(x, \gamma^{2}\right)$ $(1<x<\infty)$. Here $\gamma \rightarrow \infty$, and the results are uniformly valid in the stated intervals, $m$ and $n$ are integers, with $m$ bounded and $n$ satisfying $0 \leqslant m \leqslant n \leqslant 2 \pi^{-1} \gamma(1-\delta)$, where $\delta \in(0,1)$ is fixed. The results are obtained by an application of certain existing asymptotic solutions of differential equations, and involve elementary, Bessel, and parabolic cylinder functions. An asymptotic relationship between the prolate spheroidal equation separation parameter and the other parameters is also obtained, and error bounds are available for all approximations.

Mathematics subject classification (2010): 33E10, 34E20, 41A60.

Keywords and phrases: Spheroidal wave functions, turning point theory, WKB methods, asymptotic approximations.

\section{REFERENCES}

[1] F. M. Arscott, Periodic differential equations. An introduction to Mathieu, Lamé, and allied functions, International Series of Monographs in Pure and Applied Mathematics, 66 (A Pergamon Press Book The Macmillan Co., New York 1964 x+284 pp.)

[2] B. E. Barrowes, K. O’Neill, T. M. GrzegorczyK and J. A. Kong, On the asymptotic expansion of the spheroidal wave function and its eigenvalues for complex size parameter, Stud. Appl. Math., 113, 3 (2004), 271-301.

[3] C. J. BouWKamp, On spheroidal wave functions of order zero, J. Math. Phys. Mass. Inst. Tech., 26 (1947), 79-92.

[4] J. P. BoyD, Prolate spheroidal wavefunctions as an alternative to Chebyshev and Legendre polynomials for spectral element and pseudospectral algorithms, J. Comput. Phys., 199, 2 (2004), 688-716.

[5] W. G. C. BOYD AND T. M. Dunster, Uniform asymptotic solutions of a class of second-order linear differential equations having a turning point and a regular singularity, with an application to Legendre functions, SIAM J. Math. Anal., 17, 2 (1986), 422-450.

[6] J. R. Claycomb and Jonathan Quoc P. Tran, Introductory Biophysics: Perspectives on the Living State, Jones \& Bartlett Publishers, 2010.

[7] J. DES CloizEAUX AND M. L. MEHTA, Some asymptotic expressions for prolate spheroidal functions and for the eigenvalues of differential and integral equations of which they are solutions, J. Math. Phys., 13 (1972), 1745-1754.

[8] F. A. Dahlen And F. J. Simons, Spectral estimation on a sphere in geophysics and cosmology, Geophys. J. Int., 174, 3 (2008), 774-807.

[9] T. M. DUNSTER, Uniform asymptotic expansions for prolate spheroidal functions with large parameters, SIAM J. Math. Anal., 17, 6 (1986), 1495-1524.

[10] T. M. Dunster, Simplified asymptotic solutions of differential equations having double turning points, with an application to Legendre functions, Stud. Appl. Math., 127, 3 (2011), 250-283.

[11] P. E. Falloon, Theory and Computation of Spheroidal Harmonics with General Complex Parameters, Masters Thesis, The University of Western Australia, 2001.

[12] C. Flammer, Spheroidal Wave Functions, Stanford, CA: Stanford University Press, 1956.

[13] J. B. Jones-OliveIRA, Transient analytic and numerical results for the fluid-solid interaction of prolate spheroidal shells, J. Acoust. Soc. Amer., 99, 1 (1996), 392-407. 
[14] J. B. JONES-Oliveira AND H. R. Fischer, Absolute and uniform convergence of alternate forms of the prolate spheroidal radial wave functions, (English summary) Adv. in Appl. Math., 29 (2002), 311-327.

[15] T. Kereselidze, Z. S. Machavariani AND G. ChKAduA, Explicit spheroidal wave functions of the hydrogen atom, The European Physical Journal D, 63, 1 (2011), 81-87.

[16] S. N. Khonina, S. G. Volotovs KiĬ And V. A. SoĬFER, A method for computing the eigenvalues of prolate spheroidal functions of zero order, (Russian) Dokl. Akad. Nauk., 376 (2001), 30-32.

[17] I. V. Komarov, L. I. Ponomarev And S. Y. Slavyanov, Spheroidal and Coulomb Spheroidal Functions, (Russian) Nauka, Moscow, 1976.

[18] L. W. Li, M. S. Leong, T. S. Yeo, P. S. Kool, And K. Y. TAN, Computations of spheroidal harmonics with complex arguments: A review with an algorithm, Phys. Rev. E, 58, 5 (1998), 67926806.

[19] J. MEIXNER AND F. W. SCHÄFKE, Mathieusche Funktionen und Sphäroidfunktionen, SpringerVerlag, Berlin, 1954 (In German).

[20] J. Meixner, F. W. SchäfKe And G. Wolf, Mathieu Functions and Spheroidal Functions and Their Mathematical Foundations: Further Studies, Lecture Notes in Mathematics, 837, SpringerVerlag, Berlin-New York, 1980.

[21] J. W. Miles, Asymptotic approximations for prolate spheroidal wave functions, Studies in Appl. Math., 54 (1975), 315-349.

[22] D. Müller, B. G. Kelly and J. J. O’Brien, Spheroidal eigenfunctions of the tidal equation, Phys. Rev. Lett., 73, 11 (1994), 1557-1560.

[23] H. J. W. MÜLlER, Asymptotic expansions of prolate spheroidal wave functions and their characteristic numbers, J. Reine Angew. Math., 212 (1963), 26-48.

[24] C. Niven, On the Conduction of Heat in Ellipsoids of Revolution, Phil. Trans. R. Soc. Lond., 171 (1880), 117-151.

[25] F. W. J. Olver, Asymptotics and Special Functions, Academic Press, New York, 1974. Reprinted by AK Peters, Wellesley, 1997.

[26] F. W. J. Olver, Second-order linear differential equations with two turning points, Philos. Trans. Roy. Soc. London Ser. A, 278 (1975), 137-174.

[27] F. W. J. Olver, D. W. Lozier, R. Boisvert, C. W. Clark, (eds.), NiST Handbook of Mathematical Functions, Cambridge University Press, Cambridge, 2010. Available at http://dlmf.nist.gov/.

[28] M. K. ONG, A closed form solution of the s-wave Bethe-Goldstone equation with an infinite repulsive core, J. Math. Phys., 27, 4 (1986), 1154-1158.

[29] S. Schmutzhard, T. Hrycak And H. G. Feichtinger, A numerical study of the LegendreGalerkin method for the evaluation of the prolate spheroidal wave functions, Numer. Algorithms, 68 (2015) 691-710.

[30] B. D. SLEEMAN, Integral representations associated with high-frequency non-symmetric scattering by prolate spheroids, Quart. J. Mech. and Applied Math., 22 (1969), 405-426.

[31] D. Slepian, Some asymptotic expansions for prolate spheroidal wave functions, J. Math. Phys., 44 (1965), 99-140.

[32] D. Slepian, Some comments on Fourier analysis, uncertainty, and modeling, SIAM Rev., 25, 3 (1983), 379-393.

[33] J. A. Stratton, P. M. Morse, L. J. Chu And R. A. Hutner, Elliptic Cylinder and Spheroidal Wave Functions, John Wiley and Sons, New York, 1941.

[34] J. A. Stratton, P. M. Morse, L. J. Chu, J. D. C. Little and F. J. Corgbato, Spheroidal Wave Functions, John Wiley \& Sons, Inc., New York, NY, 1956.

[35] W. STREIFER, Uniform asymptotic expansions for prolate spheroidal wave functions, J. Math. and Phys., 47 (1968), 407-415.

[36] M. J. O. Strutt, LameÃsche, MathieÂusche und verwandte Funktionen in Physik und Technik, Ergebnisse der Mathematik und ihrer Grenzgebiete, 1, 3 (1932), Verlag Julius Springer, Berlin (In German).

[37] W. J. Thompson, Spheroidal Wave Functions, Comput. Sci. Eng., 1, 3 (1999), 84-87.

[38] G. G. Walter And X. Shen, Periodic prolate spheroidal wavelets, Numer. Funct. Anal. Optim., 26 (2005), 953-976. 
[39] G. G. WALTER AND T. SOLES KI, A new friendly method of computing prolate spheroidal wave functions and wavelets, Appl. Comput. Harmon. Anal., 19 (2005), 432-443.

[40] H. XIAO AND V. ROKHLIN, High-frequency asymptotic expansions for certain prolate spheroidal wave functions, J. Fourier Anal. Appl., 9 (2003), 575-596. 